\title{
Advances in childhood tuberculosis - contributions from the University of Cape Town
}

\author{
Heather J Zar, Brian Eley, Mark P Nicol, Anthony Figaji, Anthony Hawkridge
}

Childhood tuberculosis (TB) is common in high TB burden countries, contributing a substantial proportion to the TB caseload. The HIV epidemic has had a large impact on the incidence, diagnosis and management of childhood TB.

Aim. To review the contributions from researchers at the University of Cape Town to the field of childhood TB over the past decades.

Methods. Review of published literature on studies of childhood TB done by investigators from the University of Cape Town.

Results. Important advances have been made, especially in the areas of epidemiology, diagnosis and prevention of childhood TB. Epidemiological research has led to improved understanding of the large burden of childhood TB in Cape Town. Advances in diagnosis include use of improved specimens, particularly induced sputum and better diagnostic tests. The efficacy of GeneXpert, a rapid polymerase chain reaction (PCR)-based diagnostic test, on induced sputum specimens, has potential to enable a confirmed diagnosis in children of all ages at a range of healthcare facilities, and represents an important advance in management of children presenting with suspected TB. Advances in prevention include the establishment of a vaccine study site and several studies on immunisation, and on the use of primary isoniazid prophylaxis as an effective preventive strategy in symptomatic HIV-infected children.

Conclusion. Research in childhood TB has led to important advances in diagnosis and management, enabling better care for HIV-infected and uninfected children.

S Afr Med J 2012;102(6):518-521.
Childhood tuberculosis (TB) has increasingly been recognised as contributing a substantial proportion of the global TB caseload. In South Africa childhood TB has been estimated to contribute approximately $20 \%$ of all cases, with the most recent estimates of TB prevalence in Cape Town of 511/100 000 in children under 5 years of age. ${ }^{1}$ The resurgence in TB incidence has been driven by the HIV epidemic, with dual epidemics occurring in South Africa.

In recent decades there have been advances in several areas of childhood TB with much research conducted at the University of Cape Town. Pioneering work has been done spanning diagnosis, prevention, management and the impact of HIV. This article reviews some of these recent advances.

\section{Advances in epidemiology}

The highest TB incidence rates in the world occur in sub-Saharan African countries, many of which are also experiencing an HIV epidemic. $^{2}$ TB is a leading cause of mortality and morbidity in South Africans generally. While adult TB results from both recent and past infection, childhood $\mathrm{TB}$ results from recent infection and reflects on-going TB transmission. ${ }^{3}$ Pulmonary TB (PTB) is an important cause of morbidity and death in African children. TB in children frequently occurs in association with HIV infection. The rates of TB infection now found in South African children and adolescents are similar to those reported in Europe before chemotherapy became available. Mycobacterium tuberculosis is increasingly reported as an

Department of Paediatrics and Child Health, Red Cross War Memorial Children's Hospital, University of Cape Town

Heather J Zar, MB BCh, BC Peds (USA), BC Ped Pulm (USA), PhD

Brian Eley, MB ChB, FCP Paeds (SA), BSc Hons

Mark P Nicol, MB BCh, MMed, PhD

Anthony Figaji, $\mathrm{MB} \mathrm{ChB}, \mathrm{PhD}$

Anthony Hawkridge, MB ChB, FCPHM (SA) important cause of acute pneumonia among children from high TB prevalence areas, ${ }^{4}$ including South African children, both HIVinfected and uninfected. ${ }^{5}$

An important study by Middelkoop et al. ${ }^{3}$ in Cape Town township children under 15 years reported a $4 \%$ annual risk of infection and an annual $\mathrm{TB}$ notification rate of $0.6-1.0 \%$. The mean percentage of exposures to adult TB for TB-uninfected children, latently TB-infected children and TB cases was 5.1\%, 5.4\% and 33\% per annum and the mean number of adult smear-positive cases per exposed child was 1.0, 1.6 and 1.9, respectively. Acquisition of infection was not associated with the HIV status of the adult TB case to which the child was exposed; $36 \%$ of paediatric TB cases were diagnosed before the temporally closest adult case on their plot. They also demonstrated that the annual risk of TB infection in preschool children predominantly results from infectious residents at home, but that even with limited social interactions, much transmission occurs from non-resident adults. Reductions in household transmission could be achieved by increasing ventilation or by separating child and adult sleeping areas. ${ }^{6}$

Some important insights into the epidemiology of paediatric TB in high prevalence settings have also been gained through trials of novel TB vaccines. ${ }^{7}$ Mahomed et al. ${ }^{8}$ reviewed the clinical records of all children under 2 years of age diagnosed with TB disease in the greater Cape Town area who were born during a change in the vaccination programme from percutaneous (PC) Tokyo to intradermal (ID) Danish BCG. The annual incidence rate of $0.86-0.87 \%$ was similar for PC and ID recipients. However, the rate of disseminated TB was significantly lower in the ID cohort and significantly higher in those not vaccinated with bacilli Calmette-Guérin (BCG). ${ }^{8}$ Further analysis showed that TB incidence peaked in children aged $12-23$ months, with many children experiencing severe TB disease; $20 \%$ of children with microbiologically confirmed $\mathrm{TB}$ presented with only one feature typically associated with TB. ${ }^{9}$

Hawkridge et al..$^{10}$ in a phase IV trial comparing the efficacy of PC versus ID Tokyo BCG in the prevention of TB in 11680 newborns, demonstrated equivalence between the groups, and reported very high disease rates. From the same trial, Hatherill et al. ${ }^{11}$ demonstrated poor agreement and high variability between structured approaches 
('scoring systems') used for the screening and diagnosis of childhood TB for case yield. TB infection and disease rates in adolescents have also been studied as part of preparation for TB vaccine trials in this target population. In the Boland rural area between $40 \%$ and $60 \%$ of 13 - 18-year-olds were found to be infected, as indicated by a positive tuberculin skin test or an interferon-gamma release assay; ${ }^{12}$ the annual risk of infection was approximately $3 \%$. Disease rates, around $0.4 \%$ per annum, depending on the case definition used, appear to be lower than in infants (H Mahomed, personal communication).

\section{Advances in diagnosis}

PTB in children may be difficult to diagnose definitively as a result of nonspecific clinical and radiological signs, paucibacillary disease, and lack of capacity for microbiological diagnosis. Diagnostic uncertainty has been compounded by the HIV epidemic in which chronic lung disease, anergy and nonspecific clinical and radiological signs make definitive diagnosis even more challenging. While the clinical diagnosis has relied on chronic symptoms such as prolonged coughing or failure to thrive, recent studies have shown that TB is a cause of acute pneumonia (rather than chronic disease). In a study of children hospitalised with acute pneumonia in Cape Town, cultureconfirmed TB occurred in $8 \%$ of HIV-infected and -uninfected children. ${ }^{13}$ Clinical or radiological signs do not enable PTB to be distinguished from other pathogens. Radiological changes may be nonspecific and interpretation is further complicated by wide interand intra-observer variation in the interpretation of the presence of mediastinal lymphadenopathy, one of the major radiological features for diagnosis. ${ }^{14,15}$

\section{Immunological diagnosis}

\section{Interferon-gamma release assays (IGRAs)}

The diagnostic usefulness of IGRAs in settings characterised by high TB and HIV infection rates was explored in several studies. Either enzyme-linked immunospot (ELISPOT) or Quantiferon TB Gold (in-tube) assays were utilised in these studies. An early project in hospitalised children recorded detectable ELISPOT responses to ESAT-6 or CFP10 in 49 of 70 (70\%) children with clinical TB. Detectable responses were most frequent in children with culture-confirmed disease, i.e.10 of 12 children (83.3\%). ${ }^{16}$ Subsequent community-based studies showed that the performance of IGRAs was not superior to that of tuberculin skin tests (TSTs) for diagnosing childhood TB. The first of these studies evaluated the T-SPOT.TB assay, a commercial ELISPOT test in 218 children with a median (interquartile range) age of $18(14-24)$ months. The sensitivity of T-SPOT.TB was not statistically different from that of TST in culture-confirmed TB, and T-SPOT.TB performed more poorly when evaluated in all children with culture-confirmed and probable TB $(40 \%$ v. $52 \%) .{ }^{17}$ Another study in 397 children less than 3 years of age yielded similar sensitivities and specificities for the Quantiferon TB Gold (in-tube) assay and TST, with very good agreement between these tests. ${ }^{18}$ A similar study performed in adolescents showed that there was no significant difference in the diagnostic predictive value of Quantiferon TB Gold (in-tube) assay and TST. ${ }^{12}$

In HIV-infected children with culture-confirmed or probable TB, ELISPOT was shown to be more sensitive than TST for the detection of active TB, i.e. $25 / 39$ (64\%) v. $10 / 34$ (29\%), $p=0.005$. The sensitivity of ELISPOT in HIV-infected children was not high enough for the test to be used to rule out TB. ${ }^{19}$ Extended analysis of this dataset showed that beyond clinical findings, ELISPOT did not have statistically significant added diagnostic value in HIV-infected children with smear-negative TB.
In summary, these studies showed that there is a very limited role for IGRAs in the routine investigation of childhood TB in highburden settings. Additional study in HIV-infected children may be warranted.

\section{Tuberculin skin testing}

A study of more than 1500 children $<5$ years old who underwent tuberculin skin testing with both Mantoux and Tine tests showed that a significantly higher proportion tested positive on Tine testing $(32.8 \%$ v. $28.4 \%$ ) with reasonable agreement between the two testing methods. These findings suggested that the Tine test should be considered as a screening tool for childhood $\mathrm{TB}$ in resource-limited settings. ${ }^{20}$ Although several factors are known to influence TST response, including nutritional status, HIV infection, immunosuppressive therapy and severe primary immunodeficiencies, whether or not genetic determinants influence the response was not known until very recently. An international research collaborative explored this question by conducting a genome-wide linkage search for loci affecting TST reactivity in a large Western Cape patient cohort. Two gene loci controlling TST reactivity were identified, located on chromosomal regions $11 \mathrm{p} 14$, which influences T-cell independent resistance to $M$. tuberculosis and $5 \mathrm{p} 15$ which controls the intensity of T-cell-mediated delayed hypersensitivity to tuberculin. These findings are considered important, because further exploration of these gene loci may help to elucidate the molecular mechanisms involved in resistance to $M$. tuberculosis in high TB prevalence settings. ${ }^{21}$

\section{Microbiological diagnosis}

Definitive microbiological diagnosis and antimicrobial susceptibility have become increasingly important for reliable diagnosis in children, given the issues of pill burden, adherence and the emergence of drugresistant isolates. ${ }^{22}$ Microbiological confirmation of TB in children by culture has not been part of routine care in high-burden settings, because of the unavailability of facilities, the difficulty in obtaining samples, the poor performance of smear microscopy and the perception that microbiological yield is low. However, several studies done in Cape Town have confirmed the feasibility and usefulness of microbiological confirmation in young children with suspected PTB using sputum induction. ${ }^{23-28}$ These have shown that collection of sputum is feasible, safe and effective even in infants. Sputum induction has a number of advantages over gastric lavage (GL) as it can be done as an outpatient procedure, is relatively easy to perform, and the yield is higher. In two large studies in hospitalised infants in a tertiary care facility in South Africa (median ages 9 and 13 months), samples were successfully obtained from $95 \%$ of children. ${ }^{23,27}$ In the first study, one induced sputum (IS) sample yielded more positive cultures ( $10 \%$ of samples) than 3 sequential GL samples (6\% of samples). ${ }^{23}$ In the second study the cumulative yield from 3 IS samples (87\%) was greater than that of 3 GL samples $(65 \%, p=0.018)$, while a single IS sample was equivalent to three GL samples. ${ }^{27}$ The yield was similar in HIV-infected and -uninfected children.

Two studies in the Western Cape have evaluated the yield from sputum induction in less ill children presenting for care at primary or secondary level health facilities. ${ }^{25,26}$ Among children with mild illness admitted to a case-verification ward as part of an infant TB vaccine trial, the yield of a single IS and GL sample was equivalent; however, positive cultures (from 2 GL and 2 IS samples) were only obtained in $10 \%$ of children admitted. ${ }^{25}$ More recently a study done at a primary care clinic in Khayelitsha, investigated 270 children (median age 38 months) with suspected TB with 2 IS specimens taken on sequential days. In $11 \%$ of cases, a microbiological diagnosis 
was made; furthermore IS improved the diagnostic yield by $22 \%$, resulting in substantial numbers of children receiving treatment who would not have been treated on clinical judgement. ${ }^{26}$ Furthermore, using a Likert scale, most sputum induction procedures (91\%) were rated as very easy or easy to perform by the healthcare worker performing the test in this setting. ${ }^{26}$ Combined data from a number of studies in infants and young children involving thousands of sputum induction procedures have found IS to be safe without any serious adverse events. ${ }^{23-28}$

For prompt, effective initiation of therapy, a rapid diagnosis of TB in children is needed. Most recently, IS was reported to be highly effective for rapid detection of PTB including rifampicin resistance using Xpert MTB/RIF, an integrated specimen processing and nucleic acid amplification test for detection of $M$. tuberculosis and of resistance to rifampicin. In this study, Xpert done on two sequential sputum specimens detected $76 \%$ of children with culture-confirmed disease with $99 \%$ specificity; the yield was more than twice that of a smear. ${ }^{28}$ Detection of rifampicin resistance was highly reliable compared with line probe assay. Results, available at a median of 1 day, were much faster than culture. ${ }^{28}$ These results have provided evidence in children to support the recent recommendations by the World Health Organization (WHO) that Xpert should replace smear on sputum specimens as the initial diagnostic test in areas of high HIV prevalence or drug resistance. ${ }^{29}$

The reliability of Xpert is an important advance, enabling rapid diagnosis of childhood TB and initiation of appropriate therapy. This is important as South African studies have reported that up to $50 \%$ of children who have culture-confirmed TB are discharged from health facilities without treatment as culture results take weeks. However, upscaling of capacity for sputum induction for children in health facilities is needed, including appropriate training of staff. The tools now exist for better diagnosis of childhood TB but the operationalisation of this will depend on visionary policy makers, health managers and healthcare workers.

\section{Prevention of TB disease}

Prevention of TB disease may be achieved through a number of strategies including improvement in socio-economic living conditions, better TB control especially detection of adult cases, immunisation of children, use of isoniazid (INH) prophylaxis and use of highly active antiretroviral therapy (HAART) in HIV-infected children. Much work on understanding the immunological response to $M$. tuberculosis infection and developing improved TB vaccine strategies has been done by the South African TB Vaccine Initiative (SATVI), which is addressed in a separate publication. The use of primary INH prophylaxis in HIV-infected children is an effective strategy that has been studied in Cape Town.

\section{INH prophylaxis}

National and international guidelines have consistently recommended INH prophylaxis given for 6 months in children under 5 years of age or HIV-infected children of any age, who are exposed to a household $\mathrm{TB}$ contact or have a positive tuberculin skin test.

Recently, the efficacy and safety of primary INH prophylaxis in HIV-infected children has been shown in a randomised control trial done in Cape Town. In this study, children receiving INH had a significant reduction in TB incidence and in mortality. INH reduced the incidence of TB by $72 \%$ and mortality by $54 \%{ }^{30}$ Of note, most children were not taking HAART at the start of the study, which pre-dated antiretroviral availability in South Africa. More recently, a follow-up study of this cohort reported the efficacy of INH in addition to HAART for TB prevention. Children were followed up for a median of 5 years, during which most went onto HAART. Over this time HAART reduced the incidence of culture-confirmed TB by $70 \%$, INH alone reduced this by $80 \%$ while the combination of INH and HAART was most effective, reducing TB incidence by $90 \% .{ }^{31}$ In contrast, INH prophylaxis has not been reported to be effective for preventing TB in HIV-exposed infants who were initiated on HAART early in life and were very closely followed up, including provision of INH prophylaxis whenever there was exposure to a household TB contact. ${ }^{32}$ Differences in patient populations (clinically well HIV-exposed infants with mild immunosuppression started on HAART ${ }^{32}$ compared with HIV-infected children with advanced clinical and immunological disease $\mathrm{e}^{30,31}$ ) and study methodology, particularly very consistent and reliable use of INH prophylaxis for HIV-exposed children if there was contact with an adult TB source case, may explain these findings. ${ }^{33}$ In reality, tracing of child contacts is notoriously poorly done in TB programmes, so this strategy is unlikely to be feasible in high TB prevalence countries; moreover, this cannot be applied to children with more advanced HIV disease. Excellent long-term safety has been reported with such use of INH prophylaxis. ${ }^{34}$

Thus INH may be an effective public health intervention for HIVinfected children living in high TB prevalence areas, especially in children with more advanced HIV disease. ${ }^{35}$ Recent WHO guidelines now recommend primary INH prophylaxis in adults, adolescents and HIV-infected children over 1 year of age living in high TB prevalence areas for up to 36 months. ${ }^{36}$

\section{Tuberculous meningitis}

Tuberculous meningitis (TBM) is the most lethal form of TB, yet little progress has been made in therapy. Several controversies remain, including the treatment of increased intracranial pressure and tuberculous hydrocephalus. Although computed tomography is essential, it cannot determine intracranial pressure or distinguish between communicating and non-communicating hydrocephalus. ${ }^{37}$ This is critical to directing further therapy. Standard surgical treatment for hydrocephalus has been ventriculoperitoneal shunt insertion, but long-term complications are common. As an alternative, we described the use of endoscopy for non-communicating tuberculous hydrocephalus, a procedure in which the cerebrospinal fluid is drained internally, without relying on an external shunt. ${ }^{38}$ We reported the endoscopic features of ependymal tubercles, thickened and inflamed hypothalamus, and extensive exudates in the prepontine cistern, and a protocol for managing these difficult patients. ${ }^{39}$ Subsequent to the original description, several other centres have reported the use of endoscopy for tuberculous hydrocephalus.

Even if increased intracranial pressure is successfully treated, however, patients often die or are disabled by the vasculitis of the basal cerebral vessels and consequent brain infarction. There have been no methods to diagnose this ischaemic process or interventions to prevent it; radiology simply confirms the end result. Recently we demonstrated how brain oxygen monitoring could be used to detect ischaemia in these patients, raising the possibility of being able to intervene before infarction ensues. ${ }^{40}$ Since brain ischaemia leads to death and disability, we need to develop better invasive and noninvasive tools to enable monitoring for ischaemia.

There are major obstacles to determining which patients will deteriorate: the clinical features are nonspecific, several factors may be operative, including increased intracranial pressure, brain ischaemia, encephalopathy, seizures, and hyponatraemia. Therefore, we have embarked on a study to examine biological markers of the degree of inflammation and structural brain injury that may yield an objective measure of disease severity and a possible surrogate marker for evaluating subsequent treatment interventions. 


\section{Conclusion}

There have been major advances in the development of better diagnostic, treatment and preventive strategies for childhood TB. Prevailing perceptions that a confirmed diagnosis is difficult or unnecessary need to change as widespread implementation of new diagnostic testing and of appropriate treatment in children can potentially be achieved. However, under-recognition of the disease burden and a relative lack of resources for childhood TB remain challenging.

\section{References}

1. Wood R, Lawn SD, Caldwell J, Kaplan R, Middelkoop K, Bekker LG. Burden of new and recurrent tuberculosis in a major South African city stratifed by age and HIV-status. PLoS One 2011;6(10):e25098. 2. Zar HJ. Global paediatric pulmonology: out of Africa. Paediatr Respir Rev 2006; 7 Suppl 1:S226-228. 3. Middelkoop K, Bekker LG, Morrow C, Zwane E, Wood R. Childhood TB infection and disease: a spatial and temporal transmission analysis in a South African township. S Afr Med J 2009;99(10):738743.

4. Zar HJ. Pneumonia in HIV-infected and HIV-uninfected children in developing countries: epidemiology, clinical features, and management. Curr Opin Pulm Med 2004;10(3):176-182.

5. Gray DM, Zar HJ. Community-acquired pneumonia in HIV-infected children: a global perspective. Curr Opin Pulm Med 2010;16(3):208-216.

6. Wood R, Johnstone-Robertson S, Uys P, et al. TB transmission to young children in a South African community: modeling household and community infection risks. Clin Infect Dis 2010;51(4):401-418.

7. Hussey G, Hawkridge T, Hanekom W. Childhood TB: old and new vaccines. Paediatr Respir Rev 2007;8(2):148-154.

8. Mahomed H, Kibel M, Hawkridge T, et al. The impact of a change in bacilli Calmette-Guérin vaccine policy on TB incidence in children in Cape Town, South Africa. Pediatr Infect Dis J 2006;25(12):1167117

9. Moyo S, Verver S, Mahomed H, et al. Age-related TB incidence and severity in children under 5 years of age in Cape Town, South Africa. Int J Tuberc Lung Dis 2010;14(2):149-154.

10. Hawkridge A, Hatherill M, Little F, et al. Efficacy of percutaneous versus intradermal BCG in the prevention of TB in South African infants: randomised trial. BMJ 2008;337:a2052.

11. Hatherill M, Hanslo M, Hawkridge T, et al. Structured approaches for the screening and diagnosis of childhood TB in a high prevalence region of South Africa. Bull World Health Organ 2010;88(4):312320. Epub 29 Dec 2009.

12. Mahomed H, Hawkridge T, Verver S, et al. The tuberculin skin test versus QuantiFERON TB Gold ${ }^{\circ}$ in predicting TB disease in an adolescent cohort study in South Africa. PLoS One 2011;6(3):e17984.

13. Zar HJ, Hanslo D, Tannenbaum E, et al. Aetiology and outcome of pneumonia in human immunodeficiency virus-infected children hospitalized in South Africa. Acta Paediatr 2001;90(2):119125 .

14. Swingler GH, du Toit G, Andronikou S, van der Merwe L, Zar HJ. Diagnostic accuracy of chest radiography in detecting mediastinal lymphadenopathy in suspected pulmonary TB. Arch Dis Child 2005;90:1153-1156.

15. Andronikou S, Brauer B, Galpin J, et al. Interobserver variability in the detection of mediastinal and hilar lymph nodes on CT in children with suspected pulmonary TB. Pediatr Radiol 2006;36(4):368.

16. Nicol M, Pienaar D, Wood K, et al. Enzyme-linked immunospot assay responses to early secretory antigenic target 6 , culture filtrate protein 10 , and purified protein derivative among children with TB: implications for diagnosis and monitoring of therapy. Clin Infect Dis 2005;40:1301-1308.

17. Nicol MP, Davies MA, Wood K, et al. Comparison of T-SPOT.TB assay and tuberculin skin test for the evaluation of young children at high risk for TB in a community setting. Pediatrics 2009; 123:38-43.
18. Moyo S, Isaacs F, Gelderbloem S, et al. Tuberculin skin test and QuantiFERON*assay in young children investigated for TB in South Africa. Int J Tuberc Lung Dis 2011;15:1176-1181.

19. Davies $\mathrm{M}$, Connell T, Johannisen C, et al. Detection of TB in HIV-infected children using an enzymelinked immunospot assay. AIDS 2009; 23:961-969.

20. Pan W, Matizirofe L, Workman L, et al. Comparison of Mantoux and Tine tuberculin skin tests in BCG-vaccinated children investigated for TB. PloS One 2009;4(11):e8085.

21. Cobat A, Gallant CJ, Simkin L, et al. Two loci control tuberculin skin test reactivity in an are hyperdemic for TB. J Exp Med 2009;206:2583-2591.

22. Connell TG, Zar HJ, Nicol MP. Advances in the diagnosis of pulmonary TB in HIV-infected and HIV uninfected children. J Infect Dis 2011;204:S1151-1158.

23. Zar HJ, Tannenbaum E, Apolles P, Roux P, Hanslo D, Hussey G. Sputum induction for the diagnosi of pulmonary TB in infants and young children in an urban setting in South Africa. Arch Dis Child 2000;82(4):305-308.

24. Zar HJ, Tannenbaum E, Hanslo D, Hussey G. Sputum induction as a diagnostic tool for communityacquired pneumonia in infants and young children from a high HIV prevalence area. Pediatr Pulmonol 2003;36(1):58-62.

25. Hatherill M, Hawkridge T, Zar HJ, et al. Induced sputum or gastric lavage for community-based diagnosis of childhood pulmonary TB? Arch Dis Child 2009;94(3):195-201.

26. Moore H, de Villers PJT, Apolles P, Zar HJ. Sputum induction for diagnosis of childhood pulmonary TB (PTB) in a community setting. Int J Tuber Lung Dis (in press).

27. Zar HJ, Hanslo D, Apolles P, Swingler G, Hussey G. Induced sputum versus gastric lavage for microbiological confirmation of pulmonary TB in infants and young children: a prospective study. Lancet 2005;365:130-134

28. Nicol MP, Workman L, Isaacs W, et al. Accuracy of the Xpert MTB/RIF test for the diagnosis of pulmonary TB in hospitalized children in a high HIV-prevalence area. Lancet Infect Dis 2011;Jul 15.

29. World Health Organization (WHO). Roadmap for rolling out Xpert MTB/RIF for rapid diagnosis of TB and MDR-TB, 6 December 2010. Geneva: WHO, 2010. Available at: http://www.who.int/tb/ laboratory/roadmap_xpert_mtb-rif.pdf (accessed 4 December 2011).

30. Zar HJ, Cotton MF, Strauss S, et.al. Effect of isoniazid prophylaxis on mortality and incidence of TB in children with HIV: randomized controlled trial. BMJ 2007;334:136.

31. Frigati LJ, Kranzer K, Cotton MF, Schaaf HS, Lombard C, Zar HJ. The impact of isoniazid prophylaxis and antiretroviral therapy on TB in children infected with HIV in a high TB incidence setting. Thorax 2011;66(6):496-501.

32. Madhi SA, Nachman S, Violari A, et al. Primary isoniazid prophylaxis against TB in HIV-exposed children. N Engl J Med 2011:365:21-31.

33. Zar HJ, Lombard C. Isoniazid prophylaxis against TB in children. N Engl J Med 2011;365(16):1543.

34. Gray D, Nuttall J, Lombard C, et al. Low rates of hepatotoxicity in hiv-infected children on antiretroviral therapy with and without isoniazid prophylaxis. J Trop Pediatr 2010:56(3):159-165.

35. Gray DM, Zar H, Cotton M. Impact of TB preventive therapy on TB and mortality in HIV-infected children. Cochrane Database Syst Rev 2009;(1):CD006418.

36. WHO 2011 Guidelines for intensified TB case finding and INH preventive therapy for people living with HIV in resource constrained settings www.who.int/tb (accessed December 2011).

37. Figaji AA, Fieggen AG, Peter JC. Air encephalography for hydrocephalus in the era of neuroendoscopy. Childs Nerv Syst 2005;21:559-565.

38. Figaii AA, Fieggen AG, Peter IC. Endoscopic third ventriculostomy in tuberculous meningitis. Child Nerv Syst 2003;19:217-225

39. Figaji AA, Fieggen AG, Peter JC. Endoscopy for tuberculous hydrocephalus. Childs Nerv Syst 2007;23:79-84.

40. Figaji AA, Sandler SJ, Fieggen AG, le Roux PD, Peter JC, Argent AC. Continuous monitoring and intervention for cerebral ischemia in tuberculous meningitis. Pediatr Crit Care Med 2008;9:e25-e30.

Accepted 31 January 2012. 